\title{
NUMERICAL STUDY OF MHD FLOW AND HEAT TRANSFER OVER AN EXPONENTIALLY PERMEABLE STRETCHING SURFACE OF A CASSON FLUID
}

\section{HYMAVATHI TALLA}

Department of Applied Mathematics, Kru Dr. Mrar Pg Center, Nuzvid, Andhra Pradesh, India

ABSTRACT
Recently the study of casson fluid and its behaviour gained the attention of researchers as blood is a flow model of casson
fluid and this kind of study helps in identifying and analyzing blood flow. For MHDcasson fluid flow over a surface
which is exponentially stretched, velocity and temperature profiles were drawn for various flow parameters in particular
the effect of magnetic parameter was discussed as it has numerous industrial applications. Numerical solutions were
obtained using the Keller box process, and the consequences are presented in a graphical and thorough discussion.
KEYWORDS: Casson Fluid, Numerical Methods, Keller Box Method, Exponential Stretching Surface, Heat Transfer
\& MHD Flow

Received: Mar 29, 2020; Accepted: Apr 18, 2020; Published: Jul 07, 2020; Paper Id.: IJMPERDJUN2020229

\section{INTRODUCTION}

Many real situation problems are related to the Boundary layer flow over a stretching surface. Sakiadis studied the flow over a continuous solid sheet on boundary layer [1].L. J. Crane well-considered flow past a stretching surface[2].Heat flow over an exponentially stretching surface has extremely significant industrial applications. Based on these parameters the end product quality depends. Magyari, and. Al.[3] deliberated the transfer of heat as well as mass on flow by considering exponentially, stretching sheet. Elbashbeshy [4] reviewed the flow over a surface which is stretched exponentially. By using HAM, Sajid et al[5]and Bidin et al[6]concluded the impact of thermal radiation on boundary layer flow due to exponentially stretching sheet. Hymavathi et al[7] used Keller Box method to numerically analyze the transfer of heat of Casson fluid over an surface which is exponentially stretched.Swathy Mukhopadhyay [8] addressed the heat transfer of Casson fluid flow using shooting process.

Swathi Mukhopadhyay $[9,10]$ investigated the transfer of heat in magneto hydrodynamic flow over an exponentially stretched sheet embedded in a thermally stratified medium and the slip effects of suction / blowing and thermal radiation in magneto hydrodynamic flow over an exponentially stretching surface.In physical and theoretical dimensions of convective heat transfer Cebeci et al [11] explored in depth the technique for keller box scheme. Casson fluid is best exemplified by milk, tomato sauce, jelly, butter, human blood etc.It can be defined as a shear thinning liquid which is assumed to have infinite viscosity at zero shear rate; yield stress below which there is no flow and zero viscosity at an infinite shear rate was discussed by Dash et al[12]. Eldabe and Salwa [13] researched the flow of Casson fluid between two rotating cylinders.Boyd et al.[14] studied the steady and oscillatory blood flow of Casson fluid. Hymavathi et al [15] addressed the transfer of mass with suction and chemical reaction on Casson fluid's MHD flow. Mustafa et al [16] dealt with the flow and heat transfer by stagnation-point of a Casson fluid over a stretching surface. Bachok et al [17] investigated the heat transfer in micro polar fluid with specified heat flux over an unsteady stretching surface. 
Within the present research study, numerical solution was found to the MHD Casson fluid flow with heat transfer over a surface which is exponentially stretched is investigated. For numerical computation the Keller Box approach is discussed. Several parameters such as casson fluid, magnetic and suction / blowing parameters were tested for the velocity and temperature profiles.

\section{EQUATIONS OF MOTION AND ENERGY}

Consider an incompressible viscous fluid's boundary layer flow over a sheet which is exponentially stretched that coincides with $y=0$. The flow of the fluid is limited to $y>0$. A uniform force magnetic field B0 is applied to the stretch surface as normal.On the x-axis, two equal and opposite forces are exerted to keep the wall extended by keeping the origin fixed (see figure 1).

The rheological state equation for an incompressible Casson fluid is

$$
\tau_{j}=\left\{\begin{array}{l}
2\left(\mu_{B}+P_{y} / \sqrt{2 \pi}\right) e_{i j}, \pi>\pi_{c} \\
2\left(\mu_{B}+P_{y} / \sqrt{2 \pi_{c}}\right) e_{i j}, \pi<\pi_{c}
\end{array}\right.
$$

Here $\pi=e_{i j} e_{i j}$ and $e_{i j}$, is the $(i, j)$ th component of the deformation rate, the product of the component of the deformation rate with itself is $\pi$, the critical value of this product is $\pi_{\mathrm{c}}$ isbased on the non-Newtonian model the plastic dynamic viscosity of the non-Newtonian fluidis $\mu_{\mathrm{B}}$ andthe yield stress of the fluidois $P_{y}$.

The equations that governs the flow are

$$
\begin{aligned}
& \frac{\partial u}{\partial x}+\frac{\partial v}{\partial y}=0 \\
& u \frac{\partial u}{\partial x}+v \frac{\partial u}{\partial y}=v\left(1+\frac{1}{\beta}\right) \frac{\partial^{2} u}{\partial y^{2}}-\sigma \frac{B_{0}^{2}}{\rho} u \\
& u \frac{\partial T}{\partial x}+v \frac{\partial T}{\partial y}=k \frac{\partial^{2} T}{\partial y^{2}}
\end{aligned}
$$

Where $\mathrm{u}$ and $\mathrm{v}$ are velocity components in the directions of $x$ and $y$ respectively, $v$ is the kinematic viscosity, $\sigma$ is electrical conductivity of the fluid, $\rho$ is the fluid density (assumed constant), the parameter of Casson fluid $\beta=\mu_{B} \sqrt{2 \pi_{C}} / P_{y}$, thecoefficient of thermal diffusion of the fluid is $k$.

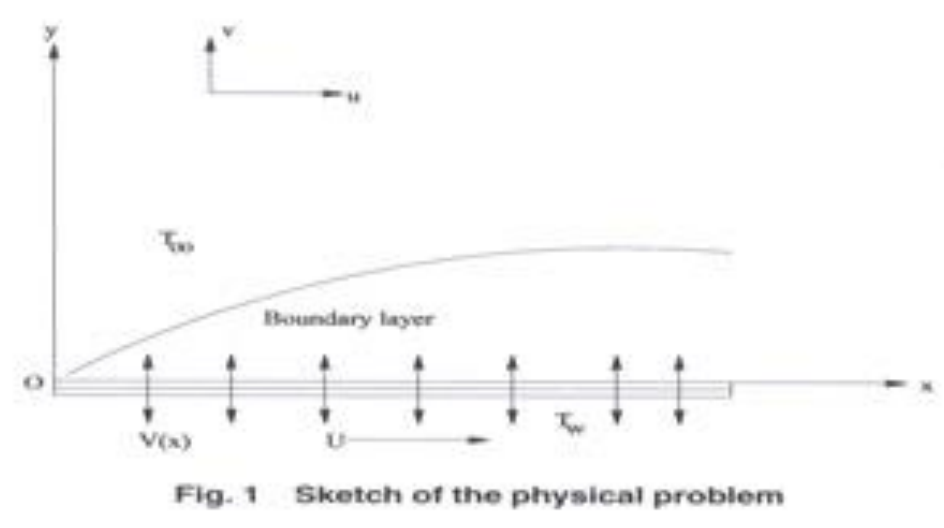




\section{Boundary}

$u=U, v=-v_{0}(x), T=T_{w}$ aty $=0$

asy $\rightarrow \infty, u \rightarrow 0, T \rightarrow T_{\infty}$

Here $U=U_{0} e^{\frac{x}{L}}$ is the stretching velocity,

$T_{w}=T_{\infty}+T_{0} e^{\frac{x}{2 L}}$ is the temperature at the sheet,

$\mathrm{U}_{0}$ and $\mathrm{T}_{0}$ are the reference velocity and temperature respectively.

$V(x)=V_{0} e^{\frac{x}{2 L}}$ is velocity at the wall with $\mathrm{V}_{0}$ as constant.

the velocity forsuction $\mathrm{V}_{0}: \mathrm{V}(\mathrm{x})>0$ and $\mathrm{V}(\mathrm{x})<0$ is the velocity for blowing.

Equations from (2)-(6) can be dimensionlessly compiled by using similarity variables

$\eta=\sqrt{\frac{U_{0}}{2 v L}} e^{\frac{x}{2 L} y}$

$U=U_{0} e^{\frac{x}{L}} f^{\prime}(\eta)$

$v=-\sqrt{\frac{v U_{0}}{2 L}} e^{\frac{x}{2 L}}\left[f(\eta)+\eta f^{\prime}(\eta)\right]$

$T=T_{\infty}+T_{0} e^{\frac{x}{2 L}} \theta(\eta)$

The chosen problem fulfils

$\left(1+\frac{1}{\beta}\right) \frac{\partial^{3} f}{\partial \eta^{3}}+\frac{\partial f}{\partial \eta} \frac{\partial^{2} f}{\partial \eta^{2}}-2\left(\frac{\partial f}{\partial \eta}\right)^{2}-M \frac{\partial f}{\partial \eta}=0(8)$

$\frac{\partial^{2} \theta}{\partial \eta^{2}}+\operatorname{Pr}\left(f \frac{\partial \theta}{\partial \eta}-\frac{\partial f}{\partial \eta} \theta\right)=0$

with respect to the boundary conditions

$\theta=1, f=S, f^{\prime}=1, a t \eta=0$,

$\theta \rightarrow 0 f^{\prime} \rightarrow 0, a s \eta \rightarrow \infty$

Where $S=v_{0} / \sqrt{\frac{U_{0} v}{2 L}}>0$ or $(<0)$ is the suction (or) blowing parameter,

$M=\frac{2 \sigma B_{0}^{2} l}{\rho U_{0} e^{x / l}}$ is the magnetic parameter and

$\operatorname{Pr}=\frac{v}{k}$ is the Prandtl number.

\section{RESULTS AND DISCUSSIONS}

By using finite difference scheme known as Keller box method,the transformed non-linear differential equations (8) and (9) 
with the boundary conditions (10) are explained by comprising the steps, consisting of the technique of finite-differences, Newton's scheme and block elimination method clearly explained by so many researchers. This approach has been applied extensively, and tends to be the most versatile compared to traditional techniques. It was described as much simpler, more programmable, more effective and easier to work.Using MATLAB, the velocity and temperature profiles are graphically plotted for different values of various parameters like Casson, Magnetic, Suction parameters and Prandtl number. It is observed that the velocity decreases as the Casson parameter increases as seen in figure 4.1 in the existence of suction / blowing.

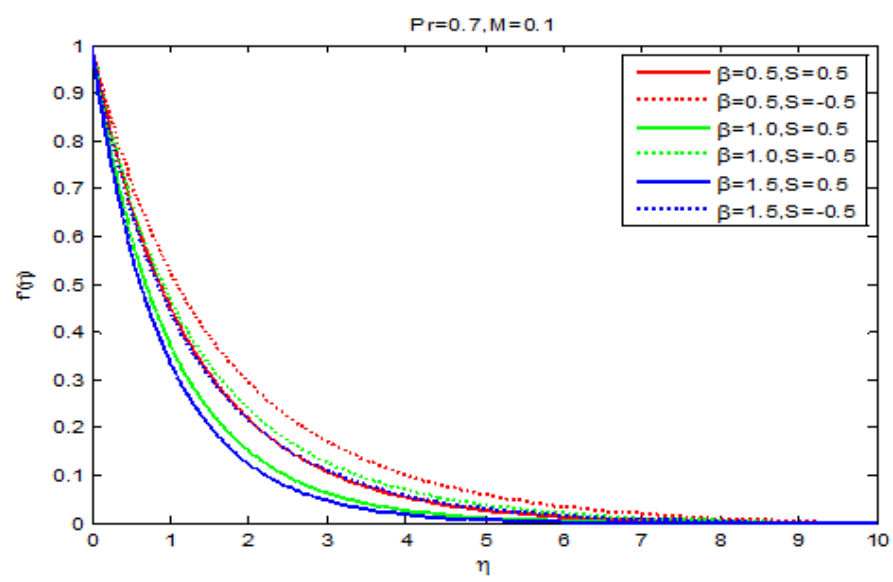

Fig.4.1. $\boldsymbol{f}^{\prime}(\boldsymbol{\eta})$ with Casson Parameter $\boldsymbol{\beta}$ in case of suction/blowing

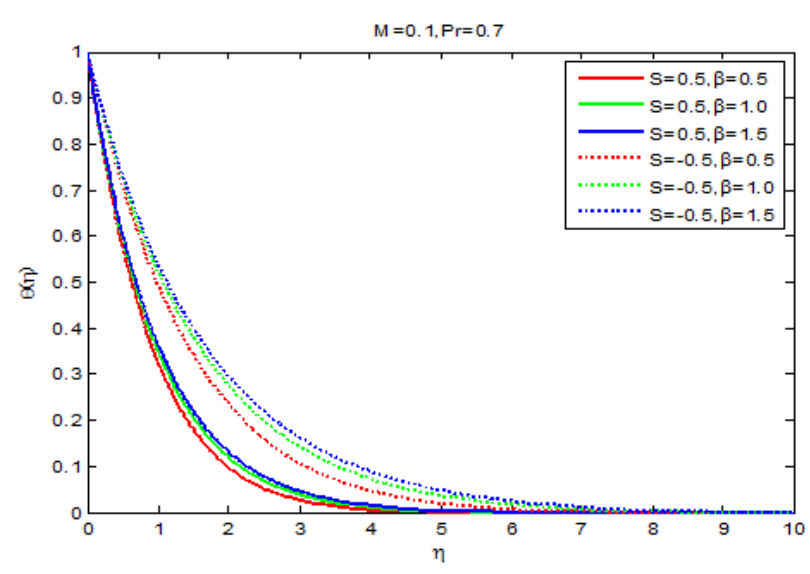

Fig.4.2. $\theta^{\prime}(\eta)$ with Casson Parameter $\beta$ in case of suction/blowing

Figure 4.2 the temperature is raising along with Casson Parameter in presence of blowing/Suction that is, in the presence of Suction / Blowing, the temperature is observed to rise with the change in Casson Parameter. 


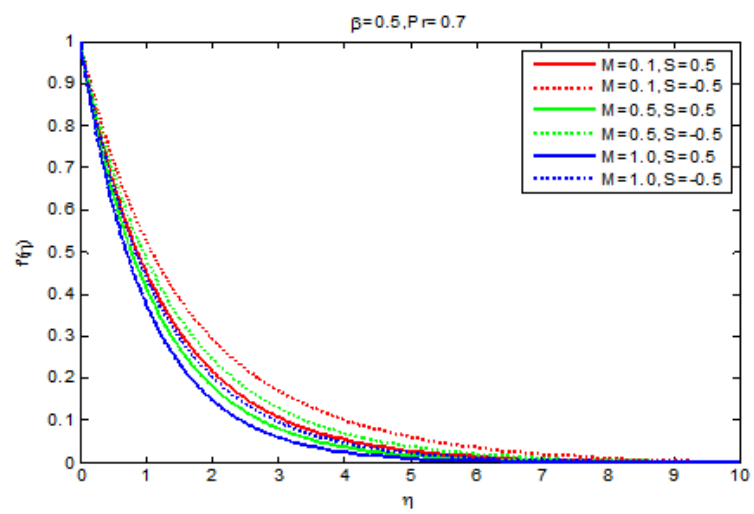

Fig.4.3. $f^{\prime}(\eta)$ with Magnetic Parameter $M$ in case of suction/blowing

The velocity is found to decline as the magnetic parameter increasesbecause of developed Lorentz force which opposes the flow as revealed in figure 4.3 in existence of suction / blowing.

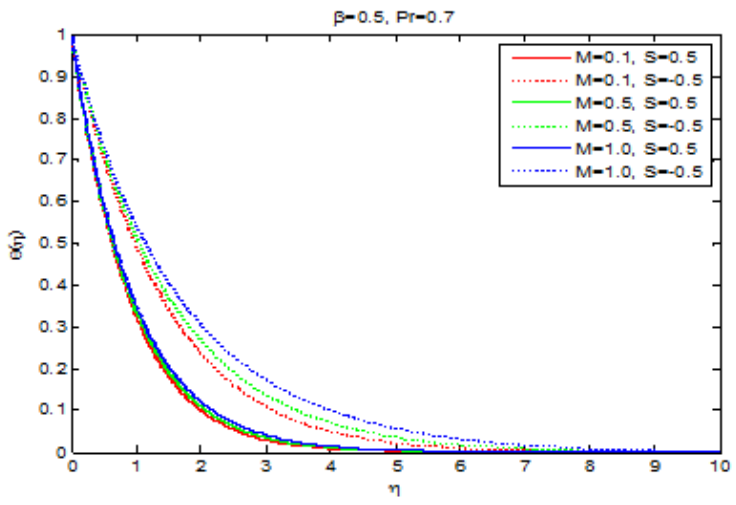

Fig.4.4. $\theta^{\prime}(\eta)$ with Magnetic Parameter $M$ in case of suction/blowing

The temperature is growing with enhancing in Magnetic parameter as shown in figure 4.4 in existence of suction / blowing.

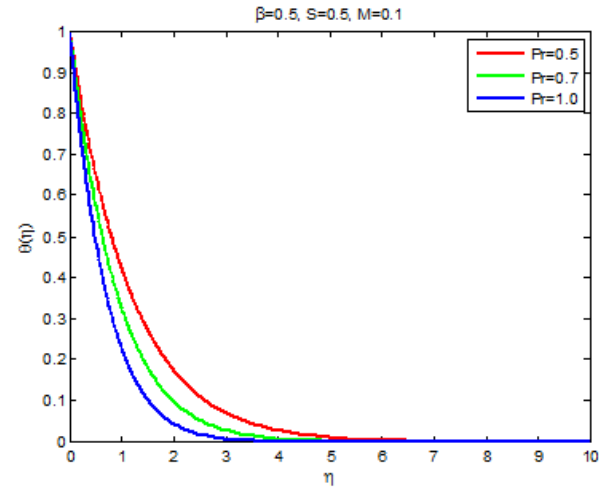

Fig.4.5. $\boldsymbol{\theta}^{\prime}(\boldsymbol{\eta})$ with Prandtl number Pr

In the presence of suction, figure 4.5 represents the effect of Prandtl number Pr on temperature profiles and found that, as Pr rises, the temperature decreases, i.e. the rise in Prdecreases the thermal boundary layer thickness. 
In presence of suction, figure 4.5 reflects the effect of the Prandtl number Pr on temperature profiles and observed that as the Prandtl number increases, then we found lessen in temperature i.e. the rise in the Prandtl number declines the thermal boundary layer thickness.

\section{CONCLUSIONS}

The present work discusses the numerical solution for the steady boundary layer flow and heat transfer study of an MHD Casson fluid over an exponentially stretching surface.It is noticed that

- Velocity and casson parameter acts opposite in working at the temperature

- $\quad$ case of suction / blowing is found to rise.

- Velocity \&magnetic parameter are inversely acting in presence of raising temperature.

- 3.Temperature decreases with rise in Pr.

- Temperature decreases with Pr increase and in turn the thickness of the thermal

- boundary layer decreases.

\section{REFERENCES}

1. Sakiadis B.C. Boundary layer bahaviour on continous solid surface: I boundary layer equation for two dimensional and axisymmetric flow.-AIChE J., vol.7, 1961, 26-28.

2. Crane, L. J., Flow Past a Stretching Plate, ZAMP, vol.21,1970, 645-647.

3. Magyari, E., and Keller, B., Heat and Mass Transfer in the Boundary Layers on an Exponentially Stretching Continuous Surface, J. Phys. D, vol.32, 1999,577-585.

4. Elbashbeshy, E. M. A., Heat Transfer Over an Exponentially Stretching Continuous Surface With Suction,Arc. Mech., vol.53, 2001, 643-651.

5. Sajid, M., and Hayat, T., Influence of Thermal Radiation on the Boundary Layer Flow Due to an Exponentially Stretching Sheet,Int. Commun. Heat Mass Transfer,vol.35,2008, 347-356.

6. Bidin, B., and Nazar, R., Numerical Solution of the Boundary Layer Flow Over an Exponentially Stretching Sheet With Thermal Radiation, Euro.J. Sci. Res., vol.33 (4), 2009, 710-717.

7. T. Hymavathi, W.Sridhar,Numerical Solution To Flow and Heat Transfer of a Casson Fluid over an exponentially permeable Stretching Surface, International Journal of Pharmacy \& Technology,vol.9(4),2017,31088-31102.

8. Swathi Mukhopadhyay, Casson Fluid Flow and Heat Transfer at an Exponentially Stretching Permeable Surface, J. AppliedMechanics, ASMEvol.80,2013,054502(1-9).

9. Swathi Mukhopadhyay, MHD boundary layer flow and heat transfer over an exponentially stretching sheet embded in a thermally stratified medium, Alexandria Engg.J.vol.52,2013,259-265

10. Swathi Mukhopadhyay, Slip effects on MHD boundary layer flow over an exponentially stretching sheet with suction/blowing and thermal radiation, Ain Shams Eng.J. 2012.

11. T. Cebeci, P. Bradshaw, Physical and computational aspects of convective heat transfer, springer-verlag, Newyork, 1988.

12. Dash, R. K., Mehta, K. N., and Jayaraman, G., Casson Fluid Flow in a Pipe Filled With a Homogeneous Porous Medium, Int. 
J. Eng. Sei., 34(10), 1996,1145-1156.

13. Eldabe, N. T. M. and Salwa, M. G. E., Heat Transfer of MHD Non- Newtonian Casson Fluid Flow Between Two Rotating Cylinders, J. Phys. Soc.Jpn., 64, 1995,41-64.

14. Boyd, J., Buick, J. M., and Green, S., Analysis of the Casson and Carreau-Yasuda Non-Newtonian Blood Models in Steady and Oscillatory Flow Using the Lattice Boltzmann Method,Phys. Fluids, vol.19,2007, 93-103.

15. T.Hymavathi, W. Sridhar, Numerical solution to mass transfer on MHD flow of Casson fluid with suction and chemical reaction, Int. Journal of Chemical Sciences vol.14(4), 2016,2183-2197.

16. Mustafa, M., Hayat, T., Pop, I. and Hendi, A. A, Stagnation-point flow and heat transfer of a Casson fluid towards a stretching sheet. Z. Naturforsch., 67a, 2012, pp.70

17. Bachok, Ishak,Nazar flow and heat transfer over an unsteady stretching sheet in a micropolar fluid with prescribed heat flux, Meccanica, vol.46(5),2011,935-942. 
